Research Article

\title{
Degree-Based Topological Indices of Polysaccharides: Amylose and Blue Starch-Iodine Complex
}

\author{
Anam Rani ${ }^{1}$ and Usman Ali $\mathbb{i D}^{2,3}$ \\ ${ }^{1}$ Department of Basic Sciences, Deanship of Preparatory Year, King Faisal University Al Ahsa, Al Hofuf, Saudi Arabia \\ ${ }^{2}$ Institute de Mathematiques de Jussieu-Paris Rive Gauche-Paris, France (Universite de Paris/Sorbonne Universite), Paris, France \\ ${ }^{3}$ CASPAM, Bahauddin Zakariya University, Multan 60000, Pakistan
}

Correspondence should be addressed to Usman Ali; uali@bzu.edu.pk

Received 2 January 2021; Revised 15 March 2021; Accepted 19 March 2021; Published 2 April 2021

Academic Editor: Muhammad Faisal Nadeem

Copyright ( $) 2021$ Anam Rani and Usman Ali. This is an open access article distributed under the Creative Commons Attribution License, which permits unrestricted use, distribution, and reproduction in any medium, provided the original work is properly cited.

\begin{abstract}
Starch is a polymer of glucose where alpha-linkages are associated with glucopyranose units. It comprises a mixture of amylose and amylopectin. Furthermore, amylose is a linear chain of hundreds of glucose molecules. Starches are not allowed to be dissolved in water. They can be digested by breaking down alpha bonds (glycosidic bonds). Its cyclic degradation products, called cyclodextrins, are the best role models for amylose. They can be considered simple turns of the amylose propeller that has imploded into a circular path. Both humans and animals have amylases, which allow them to digest starches. The important sources of starch include potatoes, rice, wheat, and maize for human consumption. The production of starches is how plants store glucose. The blue colour of starch produced by an iodine solution or iodine reaction is used for its identification. Polysaccharides with a reduced degree of polymerization, known as dextrins, are produced in the starch's partial acid hydrolysis. Complete hydrolysis leads to glucose. In this article, we compute the topological properties: Zagreb index $M_{1}(\Gamma)$ and $M_{2}(\Gamma)$, Randić index $R_{\alpha}(\Gamma)$ for $\alpha=-(1 / 2),-1,(1 / 2), 1$, atom-bond connectivity index $\mathrm{ABC}(\Gamma)$, geometric-arithmetic index $\mathrm{GA}(\Gamma)$, fourth atom-bond connectivity index $\mathrm{ABC}_{4}(\Gamma)$, fifth geometric-arithmetic index $\mathrm{GA}_{5}(\Gamma)$, and degree-based topological indices of a graph $\Gamma$ representing polysaccharides, namely, amylose and blue starch-iodine complex. In the end, we compare these indices and depict their graphic behavior.
\end{abstract}

\section{Introduction}

Amylose has the most basic structure of all nutritional polysaccharides, composed purely of glucose polymers connected only by $\alpha(1-4)$ bonds. Notice that starch is, in fact, a combination of amylose and amylopectin. Amylose is not allowed to be dissolved in water and is more difficult to digest compared to amylopectin. The complexing of amylopectin with amylose facilitates its water-another view of amylose solubility and digestibility. Amylose plays an important role in the storage of plant energy, and as plants do not require glucose to explode, its dense structure and slow breakdown features are under plant's growth. Another function of polysaccharides within cells refers to structural support. Besides, hemicelluloses are another group of polysaccharides located in plant cell walls.
In 1814, Colin and Claubry discovered the starch-iodine reaction, which is well renowned to any chemist from basic courses in qualitative and quantitative analysis.

The first topological index was derived in 1947 when Wiener worked on the boiling point of paraffin alkanes. It was known as the Wiener number. Later on, it is called a path number. The work [1] described the M-polynomial and degree-based topological indices of graphs. The authors in $[2,3]$ discussed the symmetric divisor deg index of graphs, first Zagreb after 30 years in changed form and topological indices of molecular structure. The authors in [4] also discussed the $\pi$ electron energy of hydrocarbons. In recent years, Hasni et al. computed the degree-based topological indices of the line graph of benzene ring embedded in P-type surface in the 2D network [5]. In [6], the authors calculated the index numbers for the edge version of the geometric- 
arithmetic index of nanocones. Much research has been done to explain the nature of chromophore absorption at $620 \mathrm{~nm}$ that yields starch-iodine complex, the distinctive dark blue colour. Still, there seem to have been many disputes that might be addressed to some extent in recent decades.

Let $\Gamma$ be connected simple graph with $V(\Gamma)$ a set of vertices and $E(\Gamma)$ a set of edges. Let $u \in V(\Gamma)$ and its degree is represented by $\widetilde{R_{u}}$. The idea of degree-based topological indices began from Wiener index; in 1945, Wiener defined them while studying alkane's boiling point cf. [7]. The first degree-based topological index is Randić index given by Milan Randić in [8] and is described as

$$
R_{-(1 / 2)}=\sum_{u v \in E(\Gamma)} \frac{1}{\sqrt{\widetilde{R_{u} \mathfrak{R}_{v}}}}
$$

Generalized Randić index (denoted as $R_{\alpha}(\Gamma)$ ) is described as follows:

$$
R_{\alpha}(\Gamma)=\sum_{u v \in E(\Gamma)}\left(\widetilde{\mathfrak{R}_{u}} \widetilde{\mathfrak{R}_{v}}\right)^{\alpha}, \quad \alpha=1, \frac{1}{2},-\frac{1}{2},-1 .
$$

Inverse generalized Randić index (denoted as $R R_{\alpha}(\Gamma)$ ) is described as

$$
R R_{\alpha}(\Gamma)=\sum_{u v \in E(\Gamma)} \frac{1}{\left(\sqrt{\left.\widetilde{\mathfrak{R}_{u} \widetilde{\mathfrak{R}_{v}}}\right)^{\alpha}}\right.}
$$

In $[4,9,10]$, Gutman and Trinajstić introduced and defined the first Zagreb index (denoted as $M_{1}(\Gamma)$ ) and second Zagreb index (denoted as $M_{2}(\Gamma)$ ) as

$$
M_{1}(\Gamma)=\sum_{u v \in E(\Gamma)}\left(\widetilde{\mathfrak{R}_{u}}+\widetilde{\mathfrak{R}_{v}}\right), M_{2}(\Gamma)=\sum_{u v \in E(\Gamma)}\left(\widetilde{\mathfrak{R}_{u}} \widetilde{\mathfrak{R}_{v}}\right) .
$$

In [11], Estrada introduced and studied about the atombond connectivity index (denoted as $\operatorname{ABC}(\Gamma)$ ). It is defined as follows:

$$
\operatorname{ABC}(\Gamma)=\sum_{u v \in E(\Gamma)} \sqrt{\frac{\widetilde{\mathfrak{R}_{u}}+\widetilde{\mathfrak{R}_{v}}-2}{\widetilde{\mathfrak{R}_{u} \widetilde{R}_{v}}}} .
$$

Geometric-arithmetic index (denoted as GA $(\Gamma)$ ) was given by Vukičevic cf. [12] and is defined as follows:

$$
\mathrm{GA}(\Gamma)=\sum_{u v \in E(\Gamma)} 2 \frac{\sqrt{\widetilde{\mathfrak{R}_{u} \widetilde{R}_{v}}}}{\widetilde{\mathfrak{R}_{u}}+\widetilde{\mathfrak{R}_{v}}}
$$

The fourth version of the $\mathrm{ABC}$ index (denoted as $\mathrm{ABC}_{4}(\Gamma)$ ) was introduced by Ghorbani in [13] and is defined as

$$
\mathrm{ABC}_{4}(\Gamma)=\sum_{u v \in E(\Gamma)} \sqrt{\frac{S_{u}+S_{v}-2}{S_{u} S_{v}}}
$$

where $S_{u}=\sum_{v=N_{\Gamma}(u)} \widetilde{\mathfrak{R}_{v}}$ and $N_{\Gamma}(u)=\{v \in V(\Gamma) \mid u v \in E(\Gamma)\}$.

The fifth version of the GA index (denoted as $\mathrm{GA}_{5}(\Gamma)$ ) was given by Graovac cf. [14] and is defined as

$$
\mathrm{GA}_{5}(\Gamma)=\sum_{u v \in E(\Gamma)} \frac{2 \sqrt{S_{u} S_{v}}}{S_{u}+S_{v}}
$$

\section{Result for Amylose}

Starch is a polymer of glucose whose glucopyranose alpha bonds bind cells. It is a mixture of amylose and amylopectin. Amylose is a linear chain of hundreds of glucose molecules. Starches cannot be dissolved in water. They can be digested by breaking the alpha bonds (glycosidic bonds). Amylose is a polysaccharide composed of $\alpha$-D-glucose units, linked by $\alpha(1-4)$ glycosidic bonds. It is one of the two starch components that make up about 20 to 30 percent. Due to its tight spiral structure, amylose seems to be more resilient to digestion than other starch molecules and is, thus, a significant form of resistant starch [15] (see Figure 1 for a molecular structure of amylose and Figure 2 for its unit graph and the graph model corresponding to amylose for $n=4$, where $n$ is the number of units). In amylose, there are three types of vertices having degrees 1, 2, and 3. For $n \geq 2$, amylose has four types of edge partitions as

$$
\begin{aligned}
& E_{1,2}(\Gamma)=\left\{\widetilde{\mathfrak{R}_{u}}=1, \widetilde{\mathfrak{R}_{v}}=2 \text { and } u, v \in V(\Gamma)\right\}, \\
& E_{1,3}(\Gamma)=\left\{\widetilde{\mathfrak{R}_{u}}=1, \widetilde{\mathfrak{R}_{v}}=3 \text { and } u, v \in V(\Gamma)\right\}, \\
& E_{2,3}(\Gamma)=\left\{\widetilde{\mathfrak{R}_{u}}=2, \widetilde{\mathfrak{R}_{v}}=3 \text { and } u, v \in V(\Gamma)\right\}, \\
& E_{3,3}(\Gamma)=\left\{\widetilde{\mathfrak{R}_{u}}=3, \widetilde{\mathfrak{R}_{v}}=3 \text { and } u, v \in V(\Gamma)\right\}
\end{aligned}
$$

Theorem 1. For all $n \geq 2$, let $\Gamma$ be the graph of amylose, then we have the following:

$$
\begin{aligned}
R_{1}(\Gamma) & =74 n-6, \\
R_{(1 / 2)}(\Gamma) & =29.1258 n-1.4349, \\
R_{-(1 / 2)}(\Gamma) & =5.2363 n+0.3382, \\
R_{-1}(\Gamma) & =2.4444 n+0.3334 .
\end{aligned}
$$

Proof. The general Randić connectivity index $R_{\alpha}(\Gamma)$ for $\alpha=1$ is

$$
R_{1}(\Gamma)=\sum_{u v \in E(\Gamma)} \widetilde{\mathfrak{R}_{u}} \widetilde{\mathfrak{R}_{v}}
$$

From Table 1 and equation (2), we get

$$
\begin{aligned}
R_{1}(\Gamma)= & n(1 \times 2)+(2 n+2)(1 \times 3)+(5 n-2)(2 \times 3) \\
& +4 n(3 \times 3)=74 n-6 .
\end{aligned}
$$

Now, for $\alpha=(1 / 2)$, the general Randić connectivity index $R_{\alpha}(\Gamma)$ is

$$
R_{(1 / 2)}(\Gamma)=\sum_{u v \in E(\Gamma)} \sqrt{\widetilde{\mathfrak{R}_{u} \widetilde{R}_{v}}}
$$

Again, from Table 1 and equation (2), we have 




Figure 1: Molecular structure of amylose.



(a)
From Table 1 and equation (2), we get

$$
\begin{aligned}
R_{-1}(\Gamma) & =\frac{n}{(1 \times 2)}+\frac{(2 n+2)}{(1 \times 3)}+\frac{(5 n-2)}{(2 \times 3)}+\frac{4 n}{(3 \times 3)} \\
& =2.4444 n+0.3334 .
\end{aligned}
$$

Theorem 2. For all $n \geq 2$, let $\Gamma$ be the graph of amylose, then, we have the following:

$$
\begin{aligned}
M_{1}(\Gamma) & =60 n-2, \\
M_{2}(\Gamma) & =74 n-6, \\
\operatorname{ABC}(\Gamma) & =8.5423 n+0.2188, \\
\operatorname{GA}(\Gamma) & =11.5738 n-0.2276
\end{aligned}
$$

Proof. By using Table 1 and equation (4), we get

$$
R_{-(1 / 2)}(\Gamma)=\sum_{u v \in E(\Gamma)} \frac{1}{\sqrt{\widetilde{\mathfrak{R}_{u} \widetilde{R}_{v}}}}
$$

From Table 1 and equation (2), it follows that

$$
\begin{aligned}
R_{-(1 / 2)}(\Gamma) & =\frac{n}{\sqrt{(1 \times 2)}}+\frac{(2 n+2)}{\sqrt{(1 \times 3)}}+\frac{(5 n-2)}{\sqrt{(2 \times 3)}}+\frac{4 n}{\sqrt{(3 \times 3)}} \\
& =5.2363 n+0.3382 .
\end{aligned}
$$

Now, for $\alpha=-1$, we have

$$
R_{-1}(\Gamma)=\sum_{u v \in E(\Gamma)} \frac{1}{\widetilde{\mathfrak{R}_{u}} \widetilde{\mathfrak{R}_{v}}}
$$

$$
\begin{aligned}
M_{1}(\Gamma)= & \sum_{u v \in E(\Gamma)}\left(\widetilde{\mathfrak{R}_{u}}+\widetilde{\mathfrak{R}_{v}}\right) \\
= & n(1+2)+(2 n+2)(1+3)+(5 n-2)(2+3) \\
& +4 n(3+3) \\
= & 60 n-2, \\
M_{2}(\Gamma)= & \sum_{u v \in E(\Gamma)} \widetilde{\mathfrak{R}_{u}} \widetilde{\mathfrak{R}_{v}} \\
= & n(1 \times 2)+(2 n+2)(1 \times 3)+(5 n-2)(2 \times 3) \\
& +4 n(3 \times 3) \\
= & 74 n-6 . \\
M_{2}(\Gamma)= & \sum_{u v \in E(\Gamma)} \widetilde{\mathfrak{R}_{u}} \widetilde{\mathfrak{R}_{v}} \\
= & n(1 \times 2)+(2 n+2)(1 \times 3)+(5 n-2)(2 \times 3) \\
& +4 n(3 \times 3) \\
= & 74 n-6 .
\end{aligned}
$$

By using Table 1 and equation (5), we get

$A B C(\Gamma)=\sum_{u v \in E(\Gamma)} \sqrt{\frac{\widetilde{\mathfrak{R}_{u}}+\widetilde{\mathfrak{R}_{v}}-2}{\widetilde{\mathfrak{R}_{u}} \widetilde{\mathfrak{R}}_{v}}}$

$$
\begin{aligned}
= & n \sqrt{\frac{1+2-2}{1 \times 2}}+(2 n+2) \sqrt{\frac{1+3-2}{1 \times 3}} \\
& +(5 n-2) \sqrt{\frac{2+3-2}{2 \times 3}}+4 n \sqrt{\frac{3+3-2}{3 \times 3}} \\
= & 8.5423 n+0.2188 .
\end{aligned}
$$

By using Table 1 and equation (6), we get 


$$
\begin{aligned}
G A(\Gamma)= & \sum_{u v \in E(\Gamma)} \frac{2 \sqrt{\widetilde{\mathfrak{R}_{u} \widetilde{R}_{v}}}}{\widetilde{\mathfrak{R}_{u}}+\widetilde{\mathfrak{R}_{v}}} \\
= & 2\left(n \frac{\sqrt{1 \times 2}}{(1+2)}+(2 n+2) \frac{\sqrt{1 \times 3}}{(1+3)}\right. \\
& \left.+(5 n-2) \frac{\sqrt{2 \times 3}}{(2+3)}+4 n \frac{\sqrt{3 \times 3}}{(3+3)}\right) \\
= & 11.5738 n-0.2276 .
\end{aligned}
$$

In Table 2, we give the edge partition centered on degree sum of end vertices for each edge.

Theorem 3. For all $n \geq 2$, let $\Gamma$ be the graph of amylose, then we have

$$
\begin{aligned}
\mathrm{ABC}_{4}(\Gamma) & =6.4972 n+0.2874 \\
\mathrm{GA}_{5}(\Gamma) & =11.7142 n-0.123
\end{aligned}
$$

Proof. By using Table 2 and equation (7), we get

$$
\begin{aligned}
\operatorname{ABC}_{4}(\Gamma)= & \sum_{u v \in E(\Gamma)} \sqrt{\frac{S_{u}+S_{v}-2}{S_{u} S_{v}}} \\
= & n \sqrt{\frac{2+4-2}{2 \times 4}}+\sqrt{\frac{3+6-2}{3 \times 6}}+(2 n+1) \sqrt{\frac{3+7-2}{3 \times 7}}+n \sqrt{\frac{4+7-2}{4 \times 7}}+\sqrt{\frac{6+6-2}{6 \times 6}} \\
& +(3 n-1) \sqrt{\frac{6+7-2}{6 \times 7}}+(n-1) \sqrt{\frac{6+8-2}{6 \times 8}}+(2 n+1) \sqrt{\frac{7+7-2}{7 \times 7}}+(2 n-2) \sqrt{\frac{7+8-2}{7 \times 8}} \\
= & 6.4972 n+0.2874 .
\end{aligned}
$$

By using Table 2 and equation (8), we get

$$
\begin{aligned}
\mathrm{GA}_{5}(\Gamma)= & \sum_{u v \in E(\Gamma)} \frac{2 \sqrt{S_{u} S_{v}}}{S_{u}+S_{v}} \\
= & 2\left(n \frac{\sqrt{2 \times 4}}{(2+4)}+\frac{\sqrt{3 \times 6}}{(3+6)}+(2 n+1) \frac{\sqrt{3 \times 7}}{(3+7)}+n \frac{\sqrt{4 \times 7}}{(4+7)}+\frac{\sqrt{6 \times 6}}{(6+6)}+(3 n-1) \frac{\sqrt{6 \times 7}}{(6+7)}\right. \\
& \left.+(n-1) \frac{\sqrt{6 \times 8}}{(6+8)}+(2 n+1) \frac{\sqrt{7 \times 7}}{(7+7)}+(2 n-2) \frac{\sqrt{7 \times 8}}{(7+8)}\right) \\
= & 11.7142 n-0.123 .
\end{aligned}
$$

\section{Numerical and Graphical Representation}

The numeric representation of the results calculated above is illustrated in Tables 3 and 4 , while the graphic representation is devoted to Figures 3 and 4 .

\section{Results for Blue Starch-Iodine Complex}

The main structure of amylose are cyclic degradants known as cyclodextrins. They are obtained enzymatically and may be considered as single turns of the helix of amylose imploding into a circular path. In all of these complexes, cyclodextrin molecules are positioned in front to form dimers and they are piled together to generate large cylinders that resemble the amylose helix in its global structure. The most interesting one is (trimesic acid $\left.\mathrm{H}_{2} 0\right)_{10} \mathrm{HI}_{5}$ with linear polyiodide chain. This structural model was accepted, but, unfortunately, cannot shed light on the actual configuration of the polyiodide chain (see Figure 5 for the molecular structure of blue starch-iodine and Figure 6 for its unit graph and the graph model corresponding to blue starch-iodine for $n=6$, where $n$ is the number of units). In starchiodine, there are three types of vertices having degrees 1 , 2 , and 3. For $n \geq 3$, blue starch-iodine complex has five types of edge partitions as 
TABLE 2: Edge partition based on the degree sum of end vertices of each edge.

\begin{tabular}{lccccccccc}
\hline Types of edges & $E_{\{2,4\}}$ & $E_{\{3,6\}}$ & $E_{\{3,7\}}$ & $E_{\{4,7\}}$ & $E_{\{6,6\}}$ & $E_{\{6,7\}}$ & $E_{\{6,8\}}$ & $E_{\{7,7\}}$ & $E_{\{7,8\}}$ \\
\hline Number of edges & $(2,4)$ & $(3,6)$ & $(3,7)$ & $(4,7)$ & $(6,6)$ & $(6,7)$ & $(6,8)$ & $(7,7)$ & $(7,8)$ \\
Frequency & $n$ & 1 & $2 n+1$ & $n$ & 1 & $3 n-1$ & $n-1$ & $2 n+1$ & $2 n-2$ \\
\hline
\end{tabular}

TABle 3: Numerical comparison of $M_{1}(\Gamma), M_{2}(\Gamma), \operatorname{ABC}(\Gamma), \mathrm{GA}(\Gamma), R_{1}(\Gamma), R_{-1}(\Gamma), R_{(1 / 2)}(\Gamma)$, and $R_{-(1 / 2)}(\Gamma)$.

\begin{tabular}{lccccccc}
\hline$n$ & $M_{1}(\Gamma)$ & $M_{2}(\Gamma)$ & $\mathrm{ABC}(\Gamma)$ & $\mathrm{GA}(\Gamma)$ & $R_{1}(\Gamma)$ & $R_{-1}(\Gamma)$ & $R_{(1 / 2)}(\Gamma)$ \\
\hline 1 & 58 & 68 & 8.7611 & 11.3462 & 68 & 2.7778 & 27.6909 \\
2 & 118 & 142 & 17.3034 & 22.92 & 142 & 5.2222 & 56.8166 \\
3 & 178 & 216 & 25.8457 & 34.4938 & 216 & 7.6667 & 85.9424 \\
4 & 238 & 290 & 34.388 & 46.0676 & 290 & 10.1111 & 115.0682 \\
5 & 298 & 364 & 42.9303 & 57.6414 & 364 & 12.5556 & 144.1939 \\
6 & 358 & 438 & 51.4726 & 69.2152 & 438 & 15 & 173.8109 \\
7 & 418 & 512 & 60.0149 & 80.789 & 512 & 17.4444 & 202.4455 \\
8 & 478 & 586 & 68.5572 & 92.3628 & 586 & 19.8889 & 231.5712 \\
9 & 538 & 660 & 77.0995 & 103.9366 & 660 & 22.3333 & 260.6969 \\
10 & 598 & 734 & 85.6418 & 115.5104 & 734 & 24.7778 & 289.8201 \\
\hline
\end{tabular}

TABLE 4: Numerical comparison of $\mathrm{ABC}_{4}(\Gamma)$ and $\mathrm{GA}_{5}(\Gamma)$.

\begin{tabular}{|c|c|c|c|c|c|c|c|c|c|c|}
\hline$n$ & 1 & 2 & 3 & 4 & 5 & 6 & 7 & 8 & 9 & 10 \\
\hline $\mathrm{ABC}_{4}(\Gamma)$ & 6.785 & 13.282 & 19.779 & 26.276 & 32.773 & 39.271 & 45.768 & 52.265 & 58.762 & 65.259 \\
\hline $\mathrm{GA}_{5}(\Gamma)$ & 11.591 & 23.305 & 35.019 & 46.734 & 58.448 & 70.162 & 81.876 & 93.5906 & 105.305 & 117.019 \\
\hline
\end{tabular}



(a)

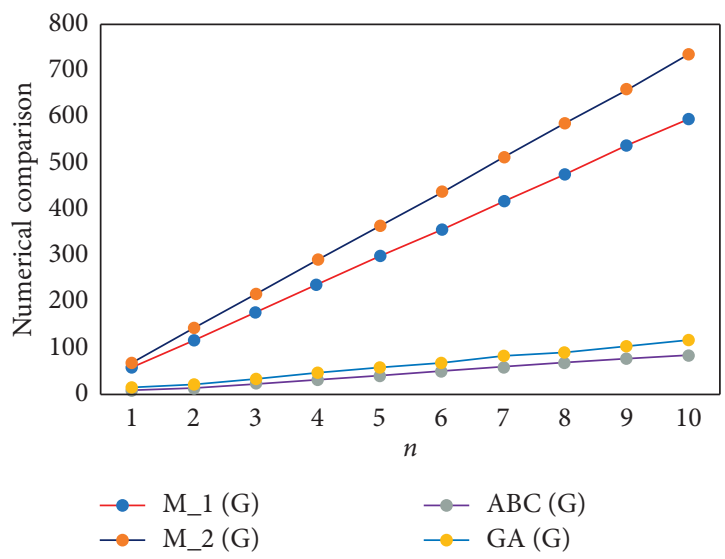

(b)

FIgure 3: (a) Comparison of $R_{\alpha}$ for $\alpha=1,-1,(1 / 2),-(1 / 2)$ and (b) comparison of $M_{1}(\Gamma), M_{2}(\Gamma), \mathrm{ABC}(\Gamma)$, and GA(Г)



Figure 4: Comparison of $\mathrm{ABC}_{4}(\Gamma)$ and $\mathrm{GA}_{5}(\Gamma)$

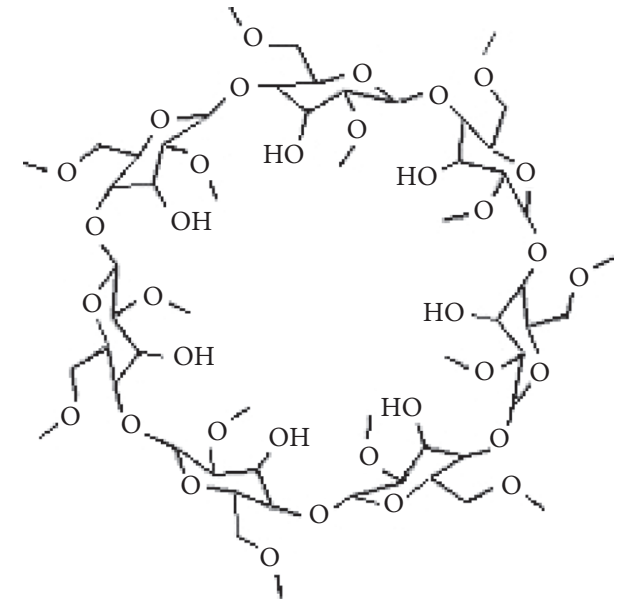

FIGURE 5: Molecular structure of blue starch-iodine. 


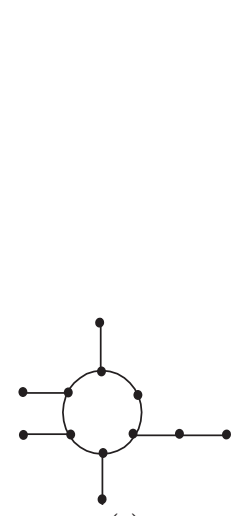

(a)



(b)
Figure 6: (a) Graph of blue starch-iodine for $n=1$ and (b) graph of blue starch-iodine for $n=6$.

$$
\begin{aligned}
& E_{1,2}(\Gamma)=\left\{\widetilde{\mathfrak{R}_{u}}=1, \widetilde{\mathfrak{R}_{v}}=2 \text { and } u, v \in V(\Gamma)\right\}, \\
& E_{1,3}(\Gamma)=\left\{\widetilde{\mathfrak{R}_{u}}=1, \widetilde{\mathfrak{R}_{v}}=3 \text { and } u, v \in V(\Gamma)\right\}, \\
& E_{2,2}(\Gamma)=\left\{\widetilde{\mathfrak{R}_{u}}=2, \widetilde{\mathfrak{R}_{v}}=2 \text { and } u, v \in V(\Gamma)\right\}, \\
& E_{2,3}(\Gamma)=\left\{\widetilde{\mathfrak{R}_{u}}=2, \widetilde{\mathfrak{R}_{v}}=3 \text { and } u, v \in V(\Gamma)\right\}, \\
& E_{3,3}(\Gamma)=\left\{\widetilde{\mathfrak{R}_{u}}=3, \widetilde{\mathfrak{R}_{v}}=3 \text { and } u, v \in V(\Gamma)\right\} .
\end{aligned}
$$

Theorem 4. For all $n \geq 3$, let $\Gamma$ be the graph of blue starchiodine complex, we have the following $R_{\alpha}(\Gamma), \alpha \in R$ :

$$
\begin{aligned}
R_{1}(\Gamma) & =39 n^{2}-n+12, \\
R_{(1 / 2)}(\Gamma) & =16.429 n^{2}-1.0354+2.8695, \\
R_{-(1 / 2)}(\Gamma) & =3.0272 n^{2}+.5585 n-0.6764, \\
R_{-1}(\Gamma) & =0.75 n^{2}+0.6944 n-0.6667 .
\end{aligned}
$$

Proof. For $\alpha=1$, the general Randić connectivity index is

$$
R_{1}(\Gamma)=\sum_{u v \in E(\Gamma)} \widetilde{\mathfrak{R}_{u}} \widetilde{\mathfrak{R}_{v}}
$$
get

From Table 5 (for edge partition) and equation (2), we

$$
\begin{aligned}
R_{1}(\Gamma)= & 2 n(1 \times 2)+\left[\sum_{i=1}^{n-1}(n+2)-2\right](1 \times 3) \\
& +n(2 \times 2)+\left[\sum_{i=1}^{n-1}(6 n-2)+2\right](2 \times 3)+4 n(3 \times 3) \\
= & 39 n^{2}-n+12 .
\end{aligned}
$$

Now, for $\alpha=(1 / 2)$, we have

$$
R_{(1 / 2)}(\Gamma)=\sum_{u v \in E(\Gamma)} \sqrt{\widetilde{\mathfrak{R}_{u}} \widetilde{\mathfrak{R}_{v}}}
$$

By using Table 5 and equation (2), after simplification, we have

$$
\begin{aligned}
R_{(1 / 2)}(\Gamma)= & 2 n \sqrt{(1 \times 2)}+\left[\sum_{i=1}^{n-1}(n+2)-2\right] \sqrt{(1 \times 3)} \\
& +n \sqrt{(2 \times 2)}+\left[\sum_{i=1}^{n-1}(6 n-2)+2\right] \sqrt{(2 \times 3)} \\
& +4 n \sqrt{(3 \times 3)} \\
= & 16.429 n^{2}-1.0354+2.8695
\end{aligned}
$$

For $\alpha=-(1 / 2)$, we have

$$
R_{-(1 / 2)}(\Gamma)=\sum_{u v \in E(\Gamma)} \frac{1}{\sqrt{\widetilde{R}_{u} \widetilde{R}_{v}}} .
$$

From Table 5 and equation (2), it follows that

$$
\begin{aligned}
R_{-(1 / 2)}(\Gamma)= & \frac{2 n}{\sqrt{(1 \times 2)}}+\frac{\left[\sum_{i=1}^{n-1}(n+2)-2\right]}{\sqrt{(1 \times 3)}} \\
& +\frac{n}{\sqrt{(2 \times 2)}}+\frac{\left[\sum_{i=1}^{n-1}(6 n-2)+2\right]}{\sqrt{(2 \times 3)}}+\frac{4 n}{\sqrt{(3 \times 3)}} \\
= & 3.0272 n^{2}+.5585 n-0.6764 .
\end{aligned}
$$

For $\alpha=-1$, we have

$$
R_{-1}(\Gamma)=\sum_{u v \in E(\Gamma)} \frac{1}{\widetilde{\mathfrak{R}_{u}} \widetilde{\mathfrak{R}_{v}}} .
$$

Again by using Table 5 and equation (2), we get

$$
\begin{aligned}
R_{-1}(\Gamma)= & \frac{2 n}{(1 \times 2)}+\frac{\left[\sum_{i=1}^{n-1}(n+2)-2\right]}{(1 \times 3)}+\frac{n}{(2 \times 2)} \\
& +\frac{\left[\sum_{i=1}^{n-1}(6 n-2)+2\right]}{(2 \times 3)}+\frac{4 n}{(3 \times 3)} \\
= & 0.75 n^{2}+0.6944 n-0.6667 .
\end{aligned}
$$

Theorem 5. For all $n \geq 3$, let $\Gamma$ be the graph of blue starchiodine complex, then we have the following:

$$
\begin{aligned}
M_{1}(\Gamma) & =34 n^{2}-2 n+4, \\
M_{2}(\Gamma) & =39 n^{2}-n+12, \\
\operatorname{ABC}(\Gamma) & =5.0591 n^{2}-0.0523 n-0.4376 \\
\operatorname{GA}(\Gamma) & =6.7448 n^{2}-3.0868 n+0.4552 .
\end{aligned}
$$

Proof. By using Table 5 and equation (4), we get 
TABle 5: Edge partition based on the degree of vertices.

\begin{tabular}{|c|c|c|c|c|c|}
\hline Types of edges & $E_{\{1,2\}}$ & $E_{\{1,3\}}$ & $E_{\{2,2\}}$ & $E_{\{2,3\}}$ & $E_{\{3,3\}}$ \\
\hline Number of edges & $(1,2)$ & $(1,3)$ & $(2,2)$ & $(2,3)$ & $(3,3)$ \\
\hline Frequency & $2 n$ & $\sum_{i=1}^{n-1}(n+2)-2$ & $n$ & $\sum_{i=1}^{n-1}(6 n-2)+2$ & $4 n$ \\
\hline
\end{tabular}

$$
\begin{aligned}
M_{1}(\Gamma)= & \sum_{u v \in E(\Gamma)}\left(\widetilde{\mathfrak{R}_{u}}+\widetilde{\mathfrak{R}_{v}}\right) \\
= & 2 n(1+2)+\left[\sum_{i=1}^{n-1}(n+2)-2\right](1+3)+n(2+2) \\
& +\left[\sum_{i=1}^{n-1}(6 n-2)+2\right](2+3)+4 n(3+3) \\
= & 34 n^{2}-2 n+4, \\
M_{2}(\Gamma)= & \sum_{u v \in E(\Gamma)} \widetilde{R}_{u} \widetilde{R}_{v} \\
= & 2 n(1 \times 2)+\left[\sum_{i=1}^{n-1}(n+2)-2\right](1 \times 3)+n(2 \times 2) \\
& +\left[\sum_{i=1}^{n-1}(6 n-2)+2\right](2 \times 3)+4 n(3 \times 3) \\
= & 39 n^{2}-n+12 .
\end{aligned}
$$

TABLE 6: Edge partition based on the degree sum of end vertices of each edge.

\begin{tabular}{lcc}
\hline Types of edges & No. of edges & Frequency \\
\hline$E_{\{2,3\}}$ & $(2,3)$ & $n$ \\
$E_{\{2,4\}}$ & $(2,4)$ & $n$ \\
$E_{\{3,5\}}$ & $(3,5)$ & $n$ \\
$E_{\{3,6\}}$ & $(3,6)$ & $\sum_{i=1}^{n-1}(1)-1$ \\
$E_{\{3,7\}}$ & $(3,7)$ & $\sum_{i=1}^{n-1}(n)-1$ \\
$E_{\{4,8\}}$ & $(4,8)$ & $n$ \\
$E_{\{5,7\}}$ & $(5,7)$ & $n$ \\
$E_{\{6,6\}}$ & $(6,6)$ & $\sum_{i=1}^{n-1}(1)-1$ \\
$E_{\{6,7\}}$ & $(6,7)$ & $n$ \\
$E_{\{6,8\}}$ & $(6,8)$ & $\sum_{i=1}^{n-1}(3 n-2)+2$ \\
$E_{\{7,7\}}$ & $(7,7)$ & $\sum_{i=1}^{n-1}(4 n-3)-2$ \\
$E_{\{7,8\}}$ & $(7,8)$ & \\
\hline
\end{tabular}

By using Table 5 and equation (5), we get

$$
\begin{aligned}
\mathrm{ABC}(\Gamma)= & \sum_{u v \in E(\Gamma)} \sqrt{\frac{\widetilde{\mathbb{R}_{u}}+\widetilde{\Re_{v}}-2}{\widetilde{R}_{u} \widetilde{R}_{v}}} \\
= & 2 n \sqrt{\frac{1+2-2}{1 \times 2}}+\left[\sum_{i=1}^{n-1}(n+2)-2\right] \sqrt{\frac{1+3-2}{1 \times 3}}+n \sqrt{\frac{2+2-2}{2 \times 2}}+\left[\sum_{i=1}^{n-1}(6 n-2)+2\right] \sqrt{\frac{2+3-2}{2 \times 3}} \\
& +4 n \sqrt{\frac{3+3-2}{3 \times 3}} \\
= & 5.0591 n^{2}-0.0523 n-0.4376 .
\end{aligned}
$$

By using Table 5 and equation (6), we get

$$
\begin{aligned}
\mathrm{GA}(\Gamma) & =\sum_{u v \in E(\Gamma)} \frac{2 \sqrt{\widetilde{\mathfrak{R}_{u}} \widetilde{\mathfrak{R}_{v}}}}{\left(\widetilde{\mathfrak{R}_{u}}+\widetilde{\mathfrak{R}_{v}}\right)} \\
& =2\left(2 n \frac{\sqrt{1 \times 2}}{(1+2)}+\left[\sum_{i=1}^{n-1}(n+2)-2\right] \frac{\sqrt{1 \times 3}}{(1+3)}+n \frac{\sqrt{2 \times 2}}{(2+2)}+\left[\sum_{i=1}^{n-1}(6 n-2)+2\right] \frac{\sqrt{2 \times 3}}{(2+3)}+4 n \frac{\sqrt{3 \times 3}}{(3+3)}\right) \\
& =6.7448 n^{2}-3.0868 n+0.4552 .
\end{aligned}
$$


TABLE 7: Numerical comparison of $M_{1}(\Gamma), M_{2}(\Gamma), \operatorname{ABC}(\Gamma), \mathrm{GA}(\Gamma), R_{1}(\Gamma), R_{-1}(\Gamma), R_{(1 / 2)}(\Gamma)$, and $R_{-(1 / 2)}(\Gamma)$.

\begin{tabular}{lcccccccc}
\hline$n$ & $M_{1}(\Gamma)$ & $M_{2}(\Gamma)$ & $\mathrm{ABC}(\Gamma)$ & $\mathrm{GA}(\Gamma)$ & $R_{1}(\Gamma)$ & $R_{-1}(\Gamma)$ & $R_{(1 / 2)}(\Gamma)$ & $R_{-(1 / 2)}(\Gamma)$ \\
\hline 1 & 36 & 50 & 4.5692 & 4.1132 & 50 & 1.361 & 18.2633 \\
2 & 136 & 166 & 19.6942 & 21.2608 & 166 & 6.0553 & 66.5148 \\
3 & 304 & 360 & 44.9374 & 51.898 & 360 & 13.4162 & 147.6243 \\
4 & 540 & 632 & 80.2988 & 96.0248 & 632 & 23.4437 & 261.5918 \\
5 & 844 & 982 & 125.7784 & 153.6412 & 982 & 36.1378 & 408.4173 \\
6 & 1216 & 1410 & 181.3762 & 224.7472 & 1410 & 51.4985 & 588.1007 \\
7 & 1656 & 1916 & 247.0922 & 309.3428 & 1916 & 69.5258 & 800.6421 \\
8 & 2164 & 2500 & 322.9264 & 407.428 & 2500 & 90.2197 & 1046.0415 \\
9 & 2740 & 3162 & 408.8788 & 519.0028 & 3162 & 113.5802 & 1324.2989 \\
10 & 3384 & 3902 & 504.9494 & 644.0672 & 3902 & 139.6073 & 1638.7891 \\
\hline
\end{tabular}

TABLE 8: Numerical comparison of $\mathrm{ABC}_{4}(\Gamma)$ and $\mathrm{GA}_{5}(\Gamma)$.

\begin{tabular}{|c|c|c|c|c|c|c|c|c|c|c|}
\hline$n$ & 1 & 2 & 3 & 4 & 5 & 6 & 7 & 8 & 9 & 10 \\
\hline $\mathrm{ABC}_{4}(\Gamma)$ & 3.35 & 14.82 & 34.45 & 62.24 & 98.19 & 142.30 & 194.57 & 255 & 323.59 & 400.34 \\
\hline $\mathrm{GA}_{5}(\Gamma)$ & 5.94 & 26.49 & 62.86 & 115.02 & 182.97 & 266.72 & 366.27 & 481.62 & 612.76 & 759.70 \\
\hline
\end{tabular}

Theorem 6. For all $n \geq 3$, let $\Gamma$ be the graph of blue starchiodine complex, we have

Proof. By using Table 6 and equation (7), we get

$$
\begin{gathered}
\operatorname{ABC}_{4}(\Gamma)=4.0798 n^{2}-0.7682 n+0.04 \\
\mathrm{GA}_{5}(\Gamma)=7.8987 n^{2}-3.1339 n+1.1727
\end{gathered}
$$

$$
\begin{aligned}
& \mathrm{ABC}_{4}(\Gamma)=\sum_{u v \in E(\Gamma)} \sqrt{\frac{S_{u}+S_{v}-2}{S_{u} S_{v}}} \\
& =n \sqrt{\frac{2+3-2}{2 \times 3}}+n \sqrt{\frac{2+4-2}{2 \times 4}}+n \sqrt{\frac{3+5-2}{3 \times 5}}+(n-2) \sqrt{\frac{3+6-2}{3 \times 6}}+\left(n^{2}-n-1\right) \sqrt{\frac{3+7-2}{3 \times 7}} \\
& +n \sqrt{\frac{4+8-2}{4 \times 8}}+n \sqrt{\frac{5+7-2}{5 \times 7}}+(n-2) \sqrt{\frac{6+6-2}{6 \times 6}}+\left(3 n^{2}-5 n+4\right) \sqrt{\frac{6+7-2}{6 \times 7}} \\
& +n \sqrt{\frac{6+8-2}{6 \times 8}}+(2 n-4) \sqrt{\frac{7+7-2}{7 \times 7}}+\left(4 n^{2}-7 n+6\right) \sqrt{\frac{7+8-2}{7 \times 8}} \\
& =4.0798 n^{2}-0.7682 n+0.04 \text {. }
\end{aligned}
$$

By using Table 6 and equation (8), we get

$$
\begin{aligned}
\mathrm{GA}_{5}(\Gamma)= & \sum_{u v \in E(\Gamma)} \frac{2 \sqrt{S_{u} S_{v}}}{\left(S_{u}+S_{v}\right)} \\
= & 2\left(n \frac{\sqrt{2 \times 3}}{(2+3)}+n \frac{\sqrt{2 \times 4}}{(2+4)}+n \frac{\sqrt{3 \times 5}}{(3+5)}+(n-2) \frac{\sqrt{3 \times 6}}{(3+6)}+\left(n^{2}-n-1\right) \frac{\sqrt{3 \times 7}}{(3+7)}+n \frac{\sqrt{4 \times 8}}{(4+8)}+n \frac{\sqrt{5 \times 7}}{(5+7)}\right. \\
& \left.+(n-2) \frac{\sqrt{6 \times 6}}{(6+6)}+\left(3 n^{2}-5 n+4\right) \frac{\sqrt{6 \times 7}}{(6+7)}+n \frac{\sqrt{6 \times 8}}{(6+8)}+(2 n-4) \frac{\sqrt{7 \times 7}}{(7+7)}+\left(4 n^{2}-7 n+6\right) \frac{\sqrt{7 \times 8}}{(7+8)}\right) \\
= & 7.8987 n^{2}-3.1339 n+1.1727 .
\end{aligned}
$$




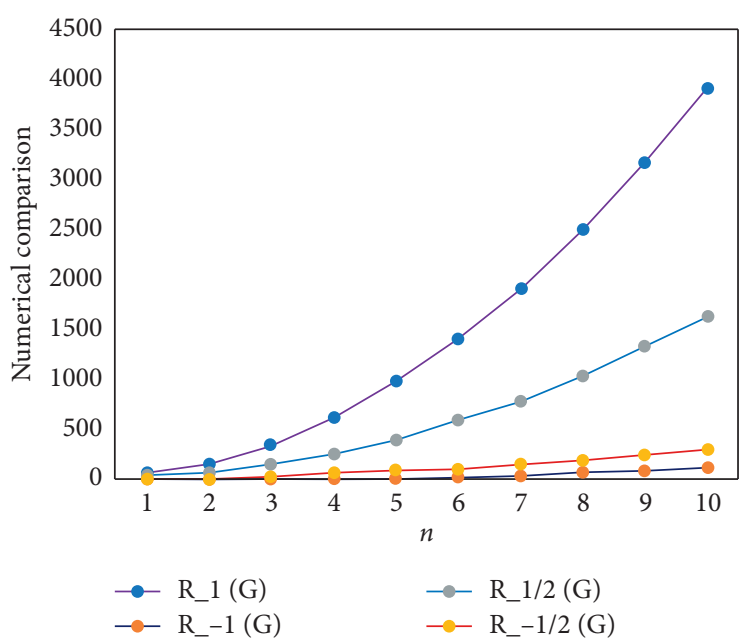

(a)

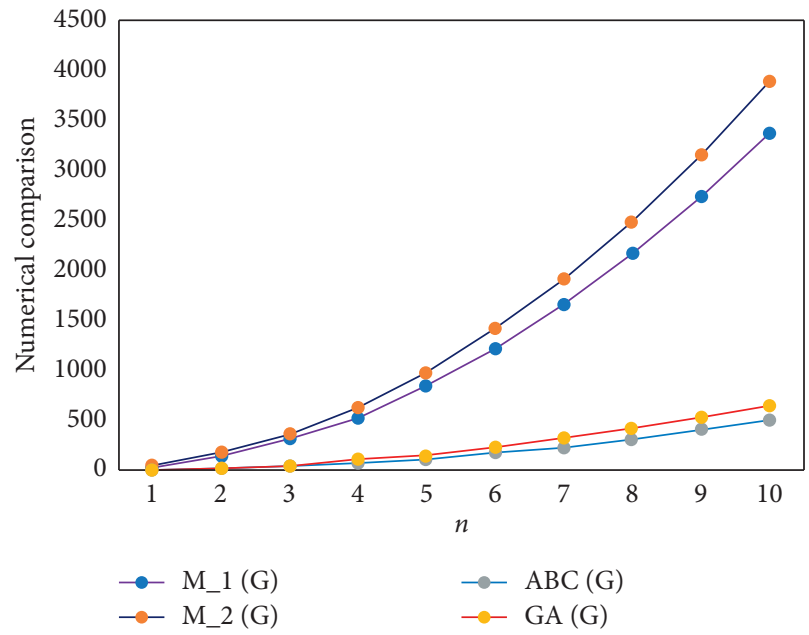

(b)

Figure 7: (a) Comparison of $R_{\alpha}(\Gamma)$ for $1,-1,(1 / 2),-(1 / 2)$ and (b) comparison of $M_{1}(\Gamma), M_{2}(\Gamma), \operatorname{ABC}(\Gamma)$, and $\mathrm{GA}(\Gamma)$.

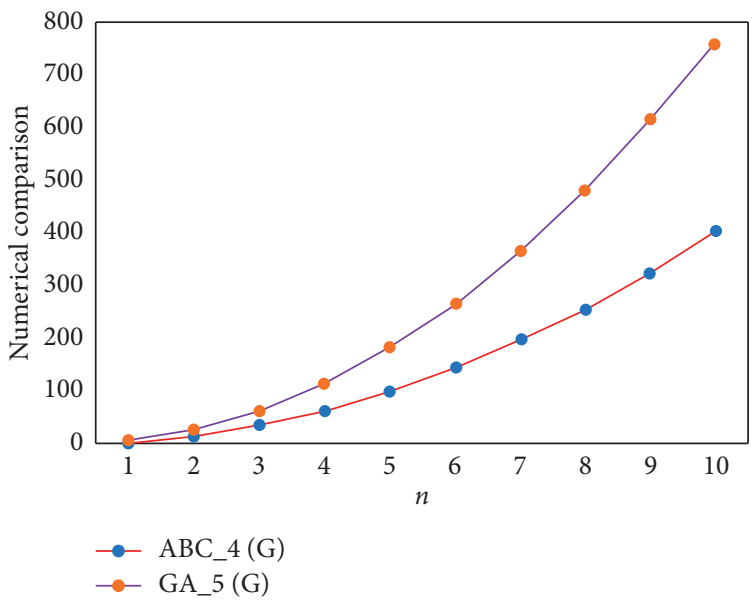

Figure 8: Comparison of $\mathrm{ABC}_{4}(\Gamma)$ and $\mathrm{GA}_{5}(\Gamma)$.

\section{Numerical and Graphical Representation}

Here, we give numeric and graphic representation for the results calculated in the above section (see Tables 7 and 8 ).

\section{Conclusion}

Amylose has a significant function in the storage of plant energy. It is not easy to digest compared to amylopectin; however, it occupies less space than amylopectin due to its spiral structure. Consequently, for storage in plants, it is the preferred starch. A mixture of iodine and potassium iodide in water is light orange-brown. When added to a sample containing starch, such as the bread pictured above, the colour will change to a deep blue (see the comparison of different indices in Figures 7 and 8 ). In this study, we have calculated degree-dependent topological indices of amylose and blue starch-iodine. We observed that $R_{(-1 / 2)}$ is closely related to geometric-arithmetic, $R_{-1}$ is closely related to atom-bond connectivity and modified atom-bond connectivity, the second Zagreb is the first Randic index, while $R_{(1 / 2)}$ is approximately equal to the modified geometric-arithmetic of amylose. Similarly, other observations can take place from the graphical representations given in this paper.

\section{Data Availability}

All kinds of data and materials, used to compute the results, are provided in Section 1.

\section{Conflicts of Interest}

The authors declare that they have no conflicts of interest.

\section{Acknowledgments}

This project was sponsored by the Deanship of Scientific Research under Nasher Proposal no. 206152, King Faisal University. 


\section{References}

[1] E. Deutsch and S. Klavžar, "M-polynomial and degree-based topological indices," Iranian Journal of Mathematical Chemistry, vol. 6, no. 2, pp. 93-102, 2015.

[2] C. Gupta, V. Lokesha, S. Shwetha, and P. Ranjini, "On the symmetric division deg index of graph," Southeast Asian Bulletin of Mathematics, vol. 40, no. 1, pp. 41-51, 2016.

[3] I. Gutman and O. Polansky, Topological Indices, Mathematical Concepts In Organic Chemistry, Springer, Berlin, Germany, 1986.

[4] I. Gutman and N. Trinajstić, "Graph theory and molecular orbitals. Total $\varphi$-electron energy of alternant hydrocarbons," Chemical Physics Letters, vol. 17, no. 4, pp. 535-538, 1972.

[5] A. Ahmad, K. Elahi, R. Hasni, and M. F. Nadeem, "Computing the degree based topological indices of line graph of benzene ring embedded in P-type-surface in 2D network," Journal of Information and Optimization Sciences, vol. 40, no. 7, pp. 1511-1528, 2019.

[6] M. F. Nadeem, S. Zafar, and Z. Zahid, "On the edge version of geomeetric-arithmetic index of nanocones," Studia Universitatis Babeş-Bolyai series Chemia, vol. 61, no. 1, pp. 273282, 2016.

[7] H. Wiener, "Structural determination of paraffin boiling points," Journal of the American Chemical Society, vol. 69, no. 1, pp. 17-20, 1947.

[8] M. Randic, "Characterization of molecular branching," Journal of the American Chemical Society, vol. 97, no. 23, pp. 6609-6615, 1975.

[9] I. Gutman and K. Das, "The first zagreb index 30 years after," MATCH Communications in Mathematical and in Computer Chemistry, vol. 50, no. 1, pp. 83-92, 2004.

[10] N. Trinajstić, S. Nikolić, A. Miličević, and I. Gutman, "About the zagreb indices," Kemija U Industriji: Časopis Kemičara I Kemijskih Inženjera Hrvatske, vol. 59, no. 12, pp. 577-589, 2010.

[11] E. Estrada, L. Torres, L. Rodríguez, and I. Gutman, “An atombond connectivity index: modelling the enthalpy of formation of alkanes," Indian Journal of Chemistry, vol. 37A, pp. 849855, 1998.

[12] D. Vucki c evi c and B. Furtula, "Topological index based on the ratios of geometrical and arithmetical means of end-vertex degree of edges," Journal of Mathematical Chemistry, vol. 46, pp. 1369-1376, 2009.

[13] M. Ghorbani and M. Hosseinzadeh, "Computing $\mathrm{ABC}_{4}$ index of nanostar dendrimers," Optoelectronics and Advanced Materials, Rapid Communications, vol. 4, pp. 1419-1422, 2010.

[14] A. Graovac, M. Ghorbani, and M. Hosseinzadeh, "Computing fifth geometric-arithmetic index for nanostar dendrimers," Journal of Mathematical Nanoscience, vol. 1, pp. 33-42, 2011.

[15] M. M. Green, G. Blankenhorn, and H. Hart, "Which starch fraction is water-soluble, Amylose or Amylopectin?" Journal of Chemical Education, vol. 52, no. 11, p. 729, 1975. 